\title{
Mechanistic study of decreased skin penetration using a combination of sonophoresis with sodium fluorescein-loaded PEGylated liposomes with D-limonene
}

\author{
This article was published in the following Dove Press journal: \\ International Journal of Nanomedicine \\ 15 December 2015 \\ Number of times this article has been viewed
}

\section{Worranan Rangsimawong \\ Praneet Opanasopit \\ Theerasak Rojanarata \\ Tanasait Ngawhirunpat}

Faculty of Pharmacy, Silpakorn University, Nakhon Pathom, Thailand

Correspondence: Tanasait Ngawhirunpat Faculty of Pharmacy, Silpakorn University, 6 Racchamakka Nai Road, Nakhon Pathom 73000, Thailand Fax +663425580 I Email ngawhirunpat_t@su.ac.th

\begin{abstract}
The effect of low frequency sonophoresis (SN, $20 \mathrm{kHz}$ ) on the skin transport of sodium fluorescein (NaFI)-loaded liposomes was investigated. An in vitro skin penetration study in open and blocked hair follicles was performed, and confocal laser scanning microscopy and scanning electron microscopy were used to visualize the penetration pathways. The results showed that SN significantly increased the flux of NaFI solution, whereas it significantly decreased the flux of NaFI-loaded polyethylene glycol-coated (PEGylated) liposomes with D-limonene (PL-LI). SN did not significantly affect the flux of NaFI-loaded conventional liposomes and PEGylated liposomes. In the blocked follicles, the flux of NaFI-loaded PL-LI both with and without SN decreased, indicating that NaFI-loaded PL-LI penetrated the skin via the transfollicular pathway. A confocal laser scanning microscopy image showed that in the skin without SN, the fluorescence intensity of NaFI-loaded PL-LI was observed in the skin and along the length of hair inside the skin, whereas in the skin with applied SN, the fluorescence intensity was detected only on the top of hair outside the skin. From scanning electron microscopy images, SN dislocated the corneocytes and reduced the deposition of PL-LI around hair follicles. These results revealed that SN may partially plug hair follicle orifices and reduce percutaneous absorption through the follicular pathway.
\end{abstract}

Keywords: sonophoresis, PEGylated liposomes, hydrophilic compound, follicular pathway

\section{Introduction}

Sonophoresis ( $\mathrm{SN})$ is a non-invasive technique for increasing the skin permeability of various medications, including hydrophilic and large molecular weight compounds such as caffeine, ${ }^{1,2}$ hydrocortisone, ${ }^{3}$ calcein, and FITC-labeled dextrans. The transdermal delivery of hydrophilic solutes with low-frequency ultrasound is likely to occur as non-specific transport across the stratum corneum (ie, both the intracellular lipid regions and the corneocytes). ${ }^{4}$ Several possible mechanisms for $\mathrm{SN}$ as a transport pathway have been suggested, such as thermal effects by absorption of ultrasound energy and cavitation effects caused by collapse and oscillation of cavitation bubbles in the ultrasound field. ${ }^{5,6}$ Cavitation has been found to be the main factor in creating aqueous pathways across the stratum corneum by distorting the lipid bilayer, which can lead to enhancing the transport of hydrophilic drugs across the skin. ${ }^{7}$

Low-frequency SN typically enhances the transport of hydrophilic molecules in solution across the skin. It can easily be coupled with other transdermal drug delivery techniques such as tape stripping, ${ }^{8}$ microneedle, ${ }^{9}$ electroporation, ${ }^{10}$ iontophoresis, ${ }^{11}$ and chemical enhancement to produce a synergistic effect on transdermal 
drug delivery through an intracellular pathway. ${ }^{12}$ The combination of SN and liposomes in skin permeation has rarely been studied, and whether these methods have a synergistic effect is still controversial. Vyas et al showed that the application of ultrasound and an ointment containing liposomes enhanced diclofenac-entrapped liposome permeation across the skin. ${ }^{13}$ However, Dahlan et al reported that the liposome application to sonicated skin prior to application of bovine serum albumin solution reduced bovine serum albumin penetration and transepidermal water loss due to the repair of sonication-induced skin disruption. ${ }^{14}$ Moreover, no mechanistic study for liposome penetration into skin combined with $\mathrm{SN}$ has yet been reported in term of skin penetration pathway, particularly for the utilization of liposomes as a transfollicular drug delivery system.

D-limonene $\left(\mathrm{C}_{10} \mathrm{H}_{16}\right)$ is one of the most common terpenes in nature that has been widely used as skin penetration enhancers. It can be remained in the lipid portion of the stratum corneum, and can fluidize or perturb the integrity of the barrier function of the stratum corneum for enhanced transport of both hydrophilic and hydrophobic drugs through the skin. ${ }^{15}$ Polyethylene glycol (PEG), a hydrophilic polymer, grafted onto the surface of the liposomes (PEGylated liposomes, PL), was reported to enhance skin penetration of zidovudine by binding to water molecules, increasing the hydration of the stratum corneum..$^{16}$ In addition, our previous work reported that D-limonene containing PL provided a synergistic effect to enhance penetration of hydrophilic compounds into and through the skin. ${ }^{17}$

Therefore, the aim of this study was to investigate the effect of low frequency $\mathrm{SN}(20 \mathrm{kHz})$ on the follicular pathway for transport of sodium fluorescein (NaFI)-loaded PL into porcine skin. PL containing D-limonene has been used as a carrier to enhance transdermal delivery of hydrophilic NaFI, with the transfollicular pathway as the major penetration pathway. ${ }^{17} \mathrm{NaFI}$ was used as a hydrophilic fluorescent compound entrapped in vesicles. Liposomal formulations were prepared by the sonication method. The particle size, shape, and in vitro skin penetration were investigated. Selectively blocked hair follicles were prepared to compare with open hair follicles. Confocal laser scanning microscopy (CLSM) and scanning electron microscopy were used to visualize the skin penetration pathways of the vesicles.

\section{Methods Materials}

Egg phosphatidylcholine (PC) and Na-salt $N$-(carbonylmethoxypolyethylen glycol-2000)-1,2-distearoyl-snglycero-3-phosphoethanolamine ( $\mathrm{PEG}_{2000}$-DSPE) were purchased from Lipoid GmbH, Ludwigshafen, Germany. Cholesterol (Chol) was purchased from Carlo Erba Reagent, Ronado, Italy. Tween 20 was purchased from Ajax Finechem, Auckland, New Zealand. NaFI and D-limonene were purchased from Sigma-Aldrich, St Louis, MO, USA. Lissamine ${ }^{\mathrm{TM}}$ rhodamine B 1,2-dihexadecanoylsn-glycero-3-phosphoethanolamine triethylammonium salt (Rh-PE) was purchased from Invitrogen, Carlsbad, CA, USA.

\section{Liposome preparation}

The formulations of the liposomes containing PC, Chol, $\mathrm{PEG}_{2000}$-DSPE, Rh-PE, NaFl, and D-limonene are shown in Table 1. The liposomes were prepared using the sonication method. Briefly, a mixture of PC, Chol, $\mathrm{PEG}_{2000}$-DSPE, and $\mathrm{Rh}-\mathrm{PE}$ (as fluorescence probe) dissolved in chloroform/ methanol $(2: 1, \mathrm{v} / \mathrm{v})$ was evaporated using the flow of $\mathrm{N}_{2}$ gas in tubes and placed in a desiccator at 6 hours. The lipid film was hydrated with NaFI solution and mixed with D-limonene in $2 \%(\mathrm{w} / \mathrm{v})$ Tween 20 solution. PL and conventional liposomes $(\mathrm{CL})$ were prepared using the same process described earlier. The particle size of all formulations was reduced following probe-sonication for 30 minutes. The particle size of all formulations was reduced following probe-sonication (Vibracell ${ }^{\mathrm{TM}}$, VCX $130 \mathrm{~PB}$, Sonics and Materials, Inc., Newtown, CT, USA) with a frequency of $40 \mathrm{kHz}$ at $40 \%$ amplitude for one cycle of 30 minutes under ice bath. An excess lipid composition was separated from vesicle formulation by centrifugation at $15,000 \mathrm{~g}$ at $4{ }^{\circ} \mathrm{C}$ for 15 minutes.

Table I The composition of the different liposomal formulations

\begin{tabular}{|c|c|c|c|c|}
\hline Formulations & $\mathrm{NaFI}(\mathrm{mM})$ & PC:Chol:PEG ${ }_{2000}$-DSPE (mM) & PC:Rh-PE (mM) & D-limonene (mM) \\
\hline$\overline{C L}$ & 5.59 & $10: 2$ & 100:1 & - \\
\hline PL & 5.59 & $10: 2: 0.12$ & 100:1 & - \\
\hline PL-LI & 5.59 & $10: 2: 0.12$ & $100: 1$ & 73.41 \\
\hline
\end{tabular}

Abbreviations: $\mathrm{CL}$, conventional liposome; Chol, cholesterol; NaFI, sodium fluorescein; PC, phosphatidylcholine; $\mathrm{PEG}_{200}$-DSPE, Na-salt N-(carbonyl-methoxypolyethylen glycol-2000)-I,2-distearoyl-sn-glycero-3-phosphoethanolamine; PL, PEGylated liposome; PL-LI, PEGylated liposome with D-limonene; Rh-PE, Lissamine ${ }^{\mathrm{TM}}$ rhodamine B I,2dihexadecanoyl-sn-glycero-3-phosphoethanolaminetriethylammonium salt. 


\section{Characterization of liposomal formulations}

\section{Particle size and surface charge}

Each liposome formulation was diluted with an appropriate amount of water and measured for particle size, size distribution, and zeta potential, using a dynamic light scattering particle size analyzer (Zetasizer Nano-ZS, Malvern Instrument, Malvern, UK) with a $4 \mathrm{~mW} \mathrm{He}-\mathrm{Ne}$ laser at a scattering angle of $173^{\circ}$.

\section{Particle size and shape by transmission electron microscopy}

Each liposome formulation was diluted with an appropriate amount of distilled water and placed in a sonicator bath for 10 minutes and then dropped onto a formvar-coated copper grid. The sample was observed using transmission electron microscopy (JEM 1230, JEOL Ltd, Tokyo, Japan) at $80 \mathrm{kV}$ for particle size and shape measurements. The image analysis software (JMicroVision V.1.2.7, University of Geneva, Geneva, Switzerland) was used to determine the diameter of the particle.

\section{Drug entrapment efficiency}

The NaFI entrapped liposomes was established by using an ultrafiltration tube with a molecular weight cutoff of 3,000 Da (Microcon YM-3; Millipore, Billerica, MA, USA). Briefly, liposomes in ultrafiltration tube were centrifuged at $4^{\circ} \mathrm{C}$ at $10,000 \times g$ for 60 minutes. $0.25 \mathrm{~mL}$ of phosphate-buffered saline (PBS) was added to the retentate and centrifuged at $4^{\circ} \mathrm{C}$ at $10,000 \times g$ for 40 minutes. The retentate was disrupted with $0.2 \mathrm{~mL}$ of $0.1 \%(\mathrm{w} / \mathrm{v})$ Triton X-100 and centrifuged at $4^{\circ} \mathrm{C}$ at $10,000 \times g$ for 10 minutes. The NaFI content of the supernatant was determined by fluorescence analysis and calculated with the following:

$$
\% \mathrm{EE}=(\mathrm{CL} / \mathrm{Ci}) \times 100
$$

where \% EE is the entrapment efficiency, $\mathrm{CL}$ is the concentration of $\mathrm{NaFI}$ in the liposomal formulation, and $\mathrm{Ci}$ is the initial concentration of NaFI added.

\section{In vitro skin permeation study Preparation of the porcine skin}

Abdominal porcine skin was taken from intrapartum stillbirth animals provided by a farm in Nakhon Pathom. Subcutaneous fat was carefully removed using medical scissors and surgical blades (thickness $\sim 0.6-0.7 \mathrm{~mm}$ ). The skin samples were frozen at $-20^{\circ} \mathrm{C}$ until use. The skin samples were thawed at room temperature using $\mathrm{PBS}(\mathrm{pH} 7.4)$ prior to the experiments.

\section{Selectively blocked hair follicle}

All hair follicles in the skin sample were blocked by using the follicular closing technique. ${ }^{15}$ Each skin sample had a hair follicle density on average between 30 and 40 follicles per application area $\left(1.96 \mathrm{~cm}^{2}\right)$. One microdrop of nail varnish using a blunt 27-gauge needle was carefully placed beside each hair follicle orifice and dried for 5 minutes to completely block the follicular shunt. Then, the skin samples were washed with PBS and mounted on Franz cells.

\section{Skin permeation study}

In vitro permeation studies of $\mathrm{NaFI}$ through porcine skin were performed using Franz diffusion cells. Briefly, $\sim 2 \mathrm{~mL}$ of NaFI-loaded liposomes was added to the skin surface in the donor compartment (an average diffusion area of $2.022 \mathrm{~cm}^{2}$ ), and the receptor compartment of the cell was filled with $6 \mathrm{~mL}$ of PBS. The skin samples, which were mounted on the Franz cells, were treated with ultrasound. The diffusion studies were performed for 24 hours. The $0.5 \mathrm{~mL}$ of receiver medium was withdrawn at predetermined time points of $1,2,4,6,8$, and 24 hours for analysis by the fluorescence-detection method, and an identical volume of PBS was added into the receiver compartment to maintain a constant volume. The cumulative amount profile was plotted against time. The steady-state flux was determined as the slope of the linear portion of the plot. Each sample was analyzed in triplicate.

For passive delivery studies, a similar procedure to the SN-treated skin studies was followed, except that the skin samples were not subjected to ultrasound treatment.

\section{Sonophoresis-treated skin}

Low frequency $\mathrm{SN}$ at $20 \mathrm{kHz}$ was generated by using an ultrasonic transducer (Vibra-cell ${ }^{\mathrm{TM}}$, VCX130 PB, Sonics and Materials, Inc.), which has a transducer probe with a radiating diameter of $6 \mathrm{~mm}$. The ultrasound transducer probe was placed inside the donor compartment with its active horn face located $3 \mathrm{~mm}$ above the skin surface. NaFI-loaded liposomes (as a coupling medium) were placed in the donor chamber. The skin was then continuously sonicated for 2 minutes (100\% duty cycle, $25 \%$ amplitude). The acoustic intensity applied was $1.90 \mathrm{~W} / \mathrm{cm}^{2}$, which was calculated from the following equation: 


$$
\begin{aligned}
\text { Intensity } & =\frac{\text { Power }(\text { Watts })}{\text { Wrea of skin }\left(\mathrm{cm}^{2}\right)} \\
& =\frac{\text { Sound energy (joules) }}{\text { Area of skin }\left(\mathrm{cm}^{2}\right) \times \text { Application time (second) }}
\end{aligned}
$$

\section{Fluorescence analysis}

The NaFI concentration was analyzed using a fluorescence spectrophotometer (Fusion ${ }^{\mathrm{TM}}$ Universal Microplate Analyzer, Packard Instrument Company, Inc., Downers Grove, IL, USA). The excitation wavelength was $485 \mathrm{~nm}$, and the emission wavelength was $535 \mathrm{~nm}$. One hundred $\mu \mathrm{L}$ of the sample was pipetted into a black 96-well plate, and fluorescence was detected for three replicates of each sample.

\section{Confocal laser scanning microscopy}

After 4 hours of in vitro skin penetration study, the whole skins were cross-sectioned using a cryostat (Leica 1850, Leica Instrument). Each skin sample was mounted on a metal sample holder using a frozen section medium (Neg50, Microm International, Waldorf, Germany). The frozen skin was sectioned into $10 \mu \mathrm{m}$ slices and placed on glass microscope slides. The skin tissues were mounted with mounting medium and covered with a cover slip. Confocal images were obtained using the $10 \times$ objective lens system of an inverted Zeiss LSM 510 META microscope (Carl Zeiss AG, Jena, Germany) with a $\mathrm{He}-\mathrm{Ne}$ laser (excitation wavelength $543 \mathrm{~nm}$; emission wavelength $580 \mathrm{~nm}$ ), Ar laser (excitation wavelength 488 $\mathrm{nm}$; emission wavelength $514 \mathrm{~nm}$ ), and diode laser (excitation wavelength $358 \mathrm{~nm}$; emission wavelength $461 \mathrm{~nm}$ ).

\section{Scanning electron microscopy}

After 4 hours in vitro skin penetration study, porcine skin was visualized to study the effect of liposomal formulations and
SN on epidermal structure. Each skin sample was cut into pieces $(1 \times 2 \mathrm{~mm})$ from the central area. The samples were rapidly frozen in liquid nitrogen and dried using a Freeze-Dry System (FreeZone 2.5; Labconco, Kansas City, MO, USA) for 24 hours. The dried specimens were gold coated using a sputtering device. Specimens were then observed with a scanning electron microscope (Camscan Mx2000; Obducat Camscan Ltd, Cambridge, UK).

\section{Statistical analysis}

One-way analysis of variance followed by a least significant difference post hoc test was used to analyze the statistical significance of observed differences. The significance level was set at $P<0.05$.

\section{Results and discussion}

\section{Physicochemical characterization of liposomal formulations}

The average size of CL, PL, and PEGylated liposomes with D-limonene (PL-LI) was 105.40 $\pm 4.50,71.30 \pm 1.22$, and $43.59 \pm 1.37 \mathrm{~nm}$, respectively, with a narrow size distribution (polydispersity index below 0.3 ). Similar to the observed particle size from transmission electron microscopy technique, the average particle size of CL, PL, and PL-LI was $112.95 \pm 14.01,58.62 \pm 6.93$, and $44.81 \pm 6.55 \mathrm{~nm}$, respectively, with a spherical shape (Figure 1). In a previous study, the particle sizes of PL and PL-LI were significantly lower than CL because PEG molecules at the surface of the liposome provided a significant reduction in attractive force (van der Waals) and an increase in the repulsive forces (steric, electrostatic, and hydration) for formation. In addition, Tween 20 added into PL-LI formulations can decrease the size of the liposome vesicle. All liposomes formulations showed a negative surface charge $(-8.74$ to $-12.65 \mathrm{mV})$. As the $\mathrm{pH}$ of
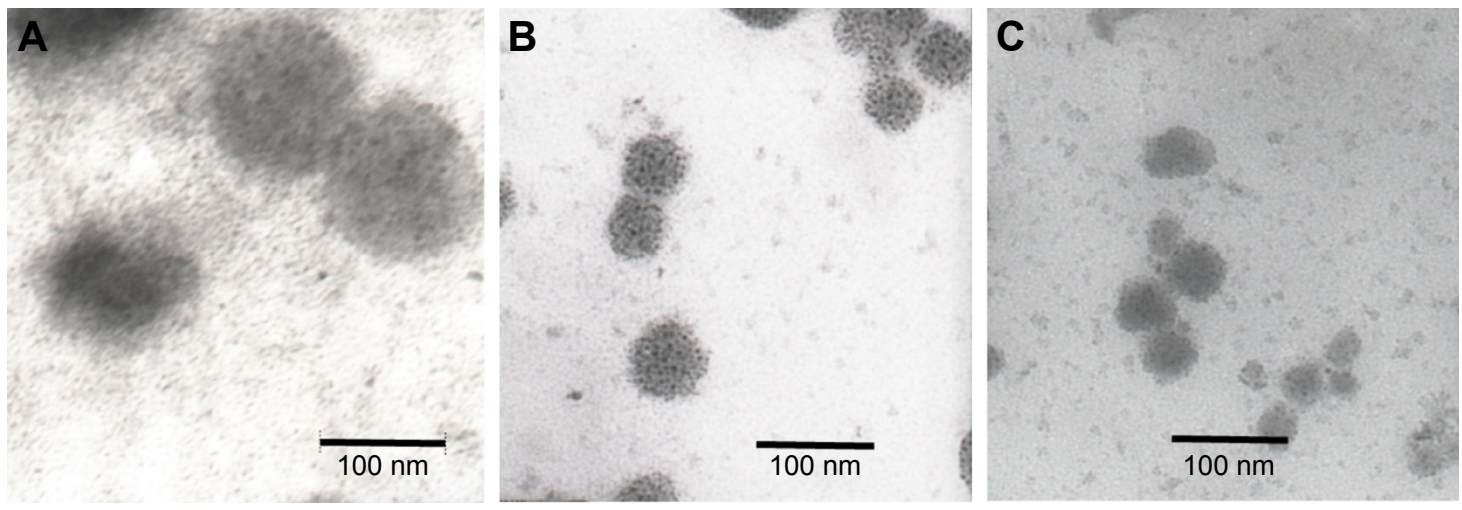

Figure I Transmission electron microscopy images of $\mathrm{NaFl}$-loaded liposomes.

Notes: (A) CL, (B) PL, and (C) PL-LI.

Abbreviations: CL, conventional liposome; NaFI, sodium fluorescein; PL, PEGylated liposome; PL-LI, PEGylated liposome with D-limonene. 
study condition ( $\mathrm{pH} 7.4$ ) was higher than the isoelectric point of PC (isoelectric point between 6 and 6.7), the PC vesicle exhibited a negative charge. ${ }^{17}$

The drug entrapment efficiency of CL, PL, and PL-LI was $17.33 \% \pm 1.13 \%, 21.76 \% \pm 1.56 \%$, and $29.60 \% \pm 2.35 \%$, respectively. According to the presence of PEG-lipids in the liposome formulation, the membrane bilayer became more polar and the efficiency of incorporation of the hydrophilic drug increased. ${ }^{16}$ In addition, edge activator and terpenes may lead to formation of pores in the bilayer, destabilize the lipid bilayers of vesicles, and increase the flexibility of the membrane. ${ }^{18-24}$

\section{In vitro skin penetration study}

Figure 2A presents the cumulative amount of NaFI permeated into the skin at different time points, in which the amount of NaFI through the skin was in the following order: PL-LI without $\mathrm{SN}>\mathrm{NaFI}$ solution with $\mathrm{SN}>\mathrm{PL}-\mathrm{LI}$ with $\mathrm{SN}>\mathrm{PL}$ without $\mathrm{SN}>\mathrm{PL}$ with $\mathrm{SN}>\mathrm{CL}$ with $\mathrm{SN}>\mathrm{CL}$ without $\mathrm{SN}>\mathrm{NaFI}$ solution without SN. For skin without SN, PL-LI showed the highest cumulative amount of NaFI, followed by PL, CL, and NaFI solution. In our previous study, liposomes containing Tween 20 (as an edge activator) increased deformability of vesicle bilayer, thus resulting in increased skin permeability of NaFI. As D-limonene (as skin penetration enhancers) was incorporated into liposomes containing an edge activator a synergistic enhancement of skin penetration of NaFI was observed. ${ }^{20}$ In SN-treated skin, the NaFI solution showed higher cumulative penetration than other formulations. However, there was no significant difference in skin permeation of NaFI-loaded CL or PL with or without SN.

From these results, the steady-state flux of NaFI was used to determine the effect of $\mathrm{SN}$ on each formulation
(Figure 2B). SN significantly increased the NaFI flux in NaFI solution from 0.0058 (without SN) to $0.2999 \mu \mathrm{g} / \mathrm{cm}^{2} / \mathrm{h}$ (with $\mathrm{SN}$ ), indicating that using $\mathrm{SN}$ resulted in a 51.7-fold enhancement in permeation over passive delivery. $\mathrm{SN}$ is a more effective technique in enhancing transdermal delivery of small hydrophilic molecules, ${ }^{2}$ as the NaFI solution permeated better through the skin with SN than with liposomal formulations. The mechanism of SN is acoustically induced cavitation to create intercellular lipid channels and defects in the stratum corneum both in the lipid bilayer and in the corneocyte, which induces aqueous permeation pathways at discrete sites. ${ }^{4,9,25}$ In $\mathrm{SN}$, both cavitation and temperature affect the solute diffusivity. Thermal energy provides a doubling of permeability for every $10^{\circ} \mathrm{C}$ of increase in temperature. ${ }^{26}$ However in this study, there was no significant rise in temperature of the donor solutions in contact with the sonicated skin (increased $\sim 1^{\circ} \mathrm{C}-2^{\circ} \mathrm{C}$ ); therefore, the NaFI diffusity was mainly due to cavitation from $\mathrm{SN}$.

In contrast, SN significantly decreased the NaFI flux of PL-LI from 0.5380 (without $\mathrm{SN}$ ) to $0.1914 \mu \mathrm{g} / \mathrm{cm}^{2} / \mathrm{h}$, indicating that using SN resulted in a 2.81-fold decrease in skin permeation compared with passive delivery. Similarly to PL, SN decreased the NaFI flux of PL from 0.0600 (without SN) to $0.0427 \mu \mathrm{g} / \mathrm{cm}^{2} / \mathrm{h}$ (1.41-fold), while SN increased the NaFI flux of CL from 0.0250 (without SN) to $0.0418 \mu \mathrm{g} / \mathrm{cm}^{2} / \mathrm{h}$ (5.36-fold). However, the NaFI flux of CL and PL between with and without SN was not significantly different, indicating that SN had no effect on the penetration route of CL and PL. According to Vyas et al, ${ }^{13}$ in the application of diclofenac-loaded liposomal ointment, with ultrasound enhancement diclofenac permeated across the skin better than liposome ointment alone. The improved diffusion is probably due to the breaking of lamellae on
A

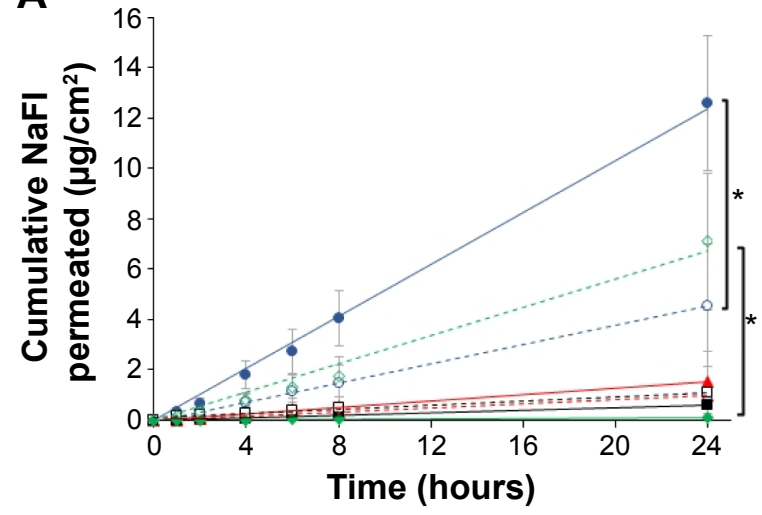

B

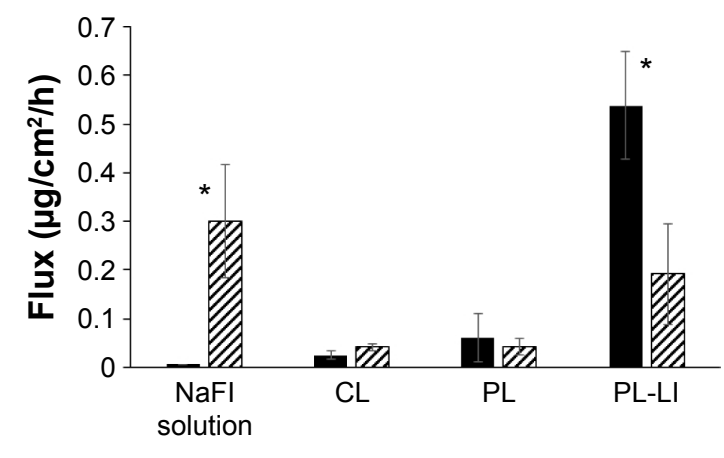

Figure 2 The skin permeation profile and flux of $\mathrm{NaFl}$ solution and NaFI loaded in different liposomal formulation with and without SN.

Notes: (A) The cumulative amount-time profiles of NaFl in different liposomal formulations with and without SN. Symbols: PL-LI with SN (O) and without SN (•), PL with $\mathrm{SN}(\triangle)$ and without SN $(\mathbf{\Delta}), \mathrm{CL}$ with SN $(\square)$ and without SN $(\square)$, and NaFl solution with $\mathrm{SN}(\diamond)$ and without $\mathrm{SN}(\diamond)$. (B) Comparison of NaFI flux $\left(\mu g / \mathrm{cm}^{2} / \mathrm{h}\right)$ of each liposome formulation with $(\mathbb{Z})$ and without $S N(\square)$. Each value represents the mean $\pm S D(n=3)$. *Indicates significant difference between groups $(P<0.05)$.

Abbreviations: CL, conventional liposome; NaFI, sodium fluorescein; PL, PEGylated liposome; PL-LI, PEGylated liposome with D-limonene; SN, sonophoresis. 
sonication. ${ }^{13}$ After releasing the entrapped drug, hydrophilic molecules were transported through the pore pathway in the stratum corneum. ${ }^{20}$ When sonication energy was terminated, phospholipid bilayer fragments were rapidly fused and closed to form liposome vesicles. ${ }^{27}$ Liposomes repair ultrasound-induced skin disruption by adsorption onto and fusion with the skin surface defect to reduce permeability. ${ }^{14}$ Moreover, the penetration pathway of PL-LI was reported to be transfollicular rather than intercellular or intracellular. ${ }^{16}$ Therefore, if ultrasound can lead to changes in the structure of the skin surface, the penetration route of NaFI-loaded PL-LI will also be affected. However, PL-LI showed higher NaFI permeation through the skin than CL and PL because D-limonene in PL-LI caused greater skin disruption than that can be repaired by liposome vesicles.

For skin penetration pathways, the delivery of the substances into the skin primarily occurs by two routes, the transfollicular route and the transepidermal routes (intercellular and intracellular penetration). ${ }^{24}$ The blocked hair follicles skin (blocked by the nail varnish) presented only the transepidermal route. Therefore, the difference in permeated flux between the open hair follicles skin (transfollicular and transepidermal route) and the blocked hair follicles skin (only transepidermal route) was calculated as the transfollicular penetration flux. ${ }^{15}$ For comparison of the blocked and open hair follicles skin, the cumulative amount and the flux of NaFI-loaded PL-LI between with SN and without SN were evaluated (Figure 3). In the blocked hair follicles skin, the flux of NaFI in PL-LI using SN and without SN was very small, thus there was no significant difference in the flux between using $\mathrm{SN}$ and without SN.
In the open hair follicle skin, skin without $\mathrm{SN}$ exhibited significantly higher cumulative amounts of NaFI than skin with SN. According to our previous study, the major penetration pathway of PL with D-limonene is the transfollicular pathway, while the intercellular and intracellular pathways are minor pathways. ${ }^{16}$ Using ultrasound leads to changes in the corneocyte layers in the uppermost layer of skin causing opening up of the continuous surface together with partially sloughing off of the hair follicle orifices, and thus the follicular route of absorption is reduced. ${ }^{3,24}$ Therefore, PL-LI that mainly permeated through the follicular route had a higher passive permeability than SN. While the flux of NaFI solution treated with $\mathrm{SN}$ and without SN was not significantly different between the open and blocked hair follicle skin (data not shown). These might be caused from that NaFI transported via transepidermal route as a major route.

\section{Visualization of fluorescence dye permeation through SN-treated skin}

CLSM images were used to visualize the fluorescence compound, NaFI-loaded liposomes and Rh-PE-probed phospholipid membrane, and demonstrate their skin penetration. Figure 4 shows cross sections of the skin after 4 hours in vitro skin permeation with and without $\mathrm{SN}$ of $\mathrm{NaFI}$ solution and NaFI-loaded-Rh-PE-labeled liposomes: CL, PL, and PL-LI. In these results, the NaFI and Rh-PE accumulated in the follicle openings, covered the hair and penetrated into the follicular duct. PL-LI without SN showed brighter fluorescence intensity of NaFI and Rh-PE in the skin and the hair follicle than other formulations.
A

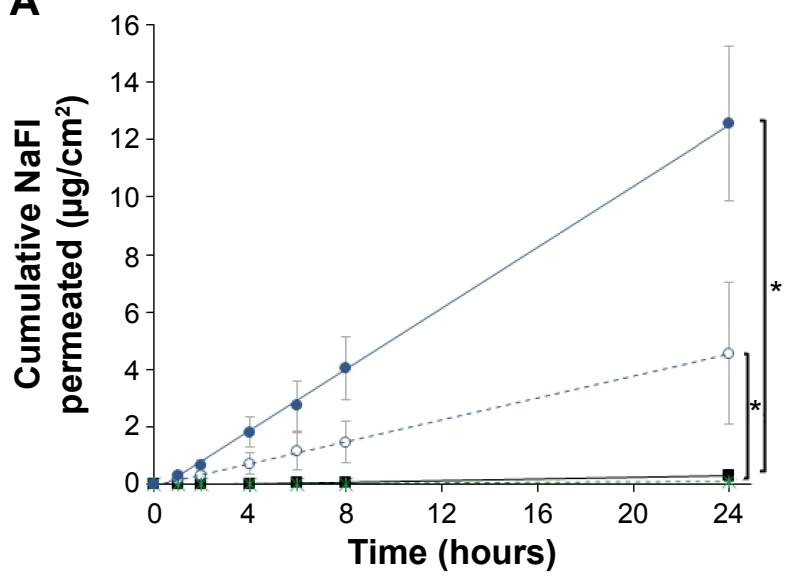

B

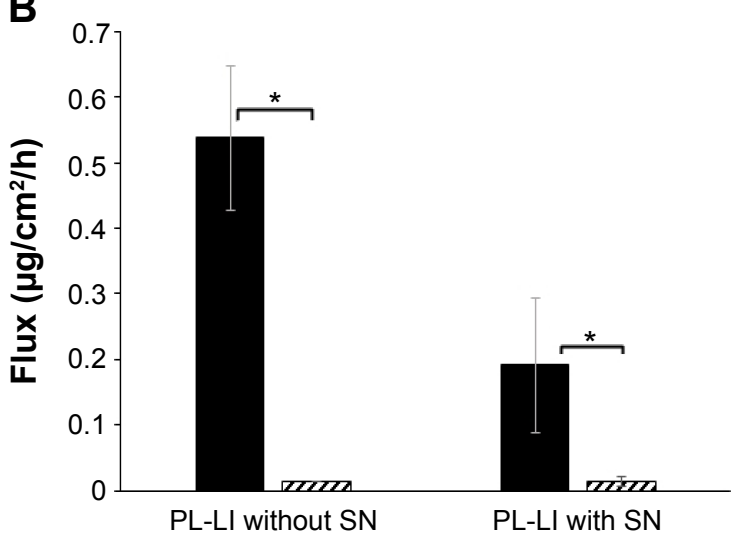

Figure 3 The skin permeation profile and flux of NaFl-loaded PL-LI through blocked hair follicles skin with SN and without SN.

Notes: (A) The skin permeation profiles of NaFl-loaded PL-LI permeated through blocked hair follicles skin (with SN [-] and without SN [*]) and open hair follicles skin

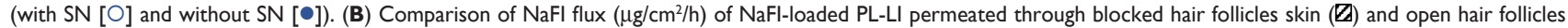
skin $(\square)$. Each value represents the mean \pm SD $(n=3)$. *Indicates significant difference from other groups $(P<0.05)$.

Abbreviations: NaFl, sodium fluorescein; PL-LI, PEGylated liposome with D-limonene; SN, sonophoresis. 


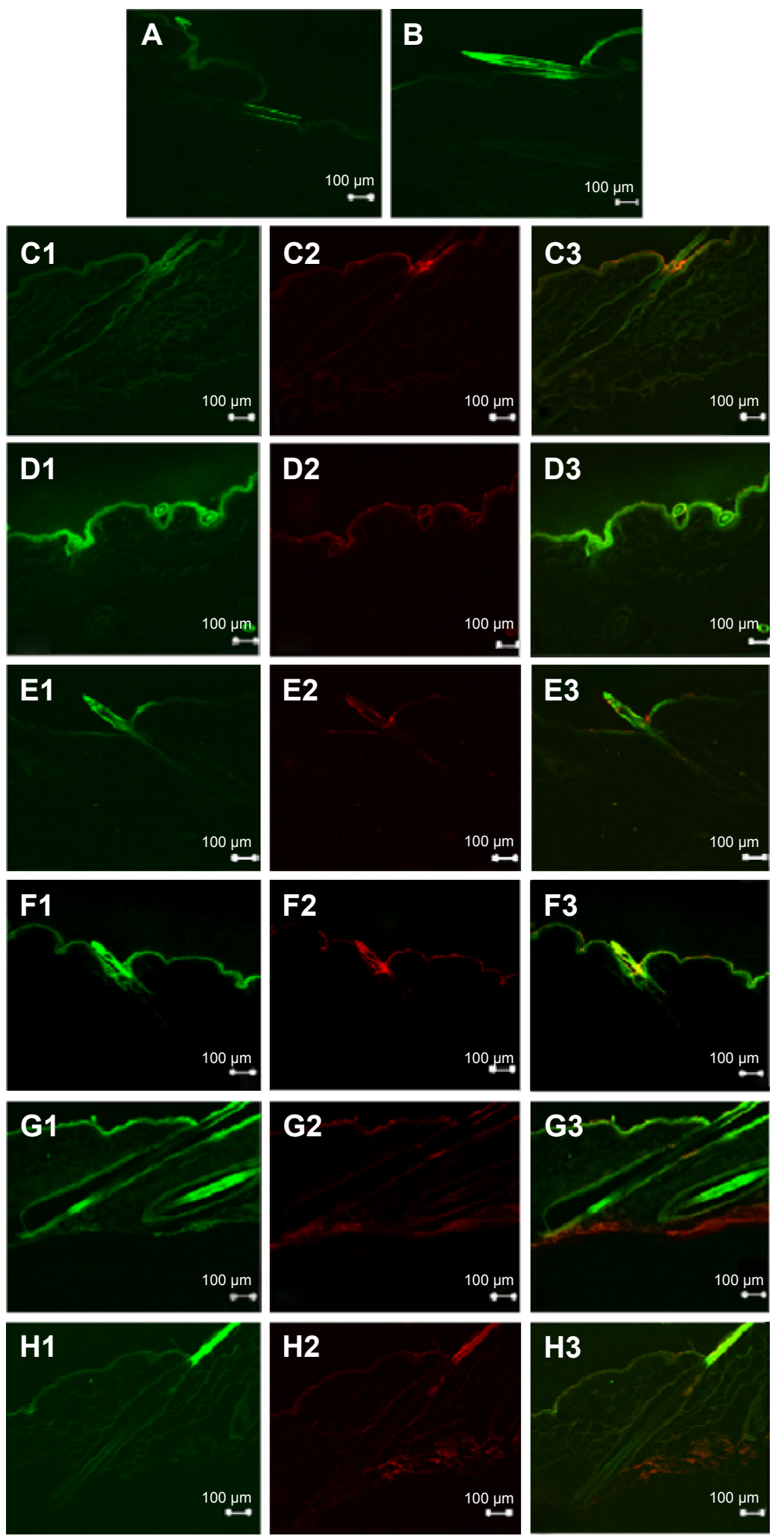

Figure 4 Confocal images of the skin cross section obtained at 4 hours after deposition of (A) NaFl solution without SN, (B) NaFI solution with SN, (C) NaFl-loadedRh-PE-labeled liposomes: CL without SN, (D) NaFI-loaded-Rh-PE-labeled liposomes: CL NaFI-loaded-Rh-PE-labeled liposomes: CL with SN, (E) NaFI-loaded-Rh-PE-labeled liposomes: PL without SN, (F) NaFI-loaded-Rh-PE-labeled liposomes: PL with SN, (G) NaFl-loaded-Rh-PE-labeled liposomes: PL-LI without SN, and (H) NaFI-loaded-Rh-PElabeled liposomes: PL-LI with SN.

Notes: In images $\mathbf{C}$ to $\mathbf{H}$, it is divided into 3 parts: I) green fluorescence of NaFl; 2) red fluorescence of Rh-PE; and 3) overlay of green fluorescence of NaFl and red fluorescence of Rh-PE. The scale bar represents $100 \mu \mathrm{m}$. All confocal images were obtained at a magnification of $\times 10$.

Abbreviations: CL, conventional liposome; NaFI, sodium fluorescein; PL, PEGylated liposome; PL-LI, PEGylated liposome with D-limonene; Rh-PE, rhodamine B I,2-dihexadecanoyl-sn-glycero-3-phosphoethanolamine triethylammonium salt; $\mathrm{SN}$, sonophoresis. 
The skin with SN of NaFI solution, CL, and PL showed brighter fluorescence intensity for both NaFI and Rh-PElabeled liposome membrane than the skin without SN. The fluorescence was deposited in the stratum corneum surface, covered the hair and in the follicle opening, but did not penetrate into the deep follicular duct. The skin image of PL-LI with SN exhibited weaker fluorescence intensity for NaFI and Rh-PE than skin without SN, which exhibited high intensity fluorescence on the top of hair outside the skin. While PL-LI without SN showed bright fluorescence intensity at a depth of $10-40 \mu \mathrm{m}$ of the stratum corneum and exhibited deeper penetration around the hair follicle orifice than other regions of skin surface, the fluorescence was also observed along the length of hair inside the skin, indicating the follicular penetration route was the main penetration route. In addition, the Rh-PE-labeled PL-LI membrane showed the deposition of fluorescence at the same region of $\mathrm{NaFI}$ and in the deepest layer of skin, suggesting that the intact vesicles might penetrate into skin. However, the fluorescence of NaFI was observed in only some parts of the skin, suggesting that some vesicles might release the entrapped drug before attaching to any part of the skin. These results indicated three mechanisms of skin penetration for the NaFI-loaded PL-LI: i) penetration associated with the liposomal bilayer (intact vesicles), ii) penetration associated with a liposomal bilayer fragment, or iii) penetration solitarily. ${ }^{28}$

The fluorescence intensity of NaFI-loaded-Rh-PE-labeled PL-LI in skin decreased when using SN, indicating that the mechanical effect of ultrasound changed the transport pathway of drug-loaded lipid vesicles. Morimoto et al reported that the differences in the physicochemical properties of the solutes, such as lipophilicity or hydrophilicity, may be affected when using low frequency ultrasound, as the ultrasound increases water transport across the skin. Thus, the distribution of more lipophilic compounds may not be influenced, ${ }^{4}$ indicating that PL-LI as a lipid vesicle carrier might not penetrate through hydrophilic transport routes in the intercellular space of the stratum corneum. In addition, liposomes adsorbed onto the skin damage caused by sonication might cause the high fluorescence intensity of both NaFI and Rh-PE-labeled CL and PL at the top of the skin layer.

\section{Scanning electron microscopy}

Figure 5 shows scanning electron micrographs of the skin surface for control (PBS), CL, PL, and PL-LI with and without SN. The surface of skin without SN was observed to assess the effect of SN on the transport route of NaFI-loaded liposomal formulations across the skin. Without SN, the surface of the stratum corneum remained relatively flat, intact, and confluent. However, in skin with SN, the corneocytes were lifted up and exhibited crack-like structures. The lowfrequency $\mathrm{SN}$ induces disruption of the structure of stratum corneum lipid bilayers and enhances skin permeability for hydrophilic molecules in solution into the viable epidermis through an intracellular pathway. ${ }^{4,8}$ However, the combination of CL or PL with SN for treated skin showed small corneocytes lifting, indicating that the lipid membrane of liposomes could fill and cover the skin damage. Although liposomes can repair the skin damage, the combination of using a chemical penetration enhancer (D-limonene) in a liposomal formulation (PL-LI) and SN resulted in greater disruption of the skin stratum corneum so the skin damage could not be repaired by liposomes. ${ }^{13,29}$

Figure 6 shows scanning electron micrographs of the skin surface view at the follicular region of the control (PBS), CL, PL, and PL-LI with and without SN. In the absence of SN, only PL-LI was clearly found to be deposited on the top of hair follicles that covered hair follicle orifices (Figure 6G, white arrow), indicating the use of the follicular route as a major penetration route. Therefore, SN had a lower amount of NaFI permeate through the skin than without SN (Figure 2) because the ultrasound changes the ultrastructure of the stratum corneum, dislocating the top layer of corneocytes. Additionally, the follicles constitute a very tiny fraction $(\leq 0.1 \%$ approximately) of the total skin surface area; therefore, many detached corneocytes might partially slough off the hair follicle opening, ${ }^{3}$ closing the main penetration pathway of NaFI-loaded-PL-LI. Therefore, the partial plugging of the hair follicle orifices caused by ultrasound energy altering the ultrastructure of stratum corneum might reduce the absorption of NaFI and PL-LI via the follicular route. ${ }^{30}$

\section{Conclusion}

This work demonstrated that low frequency SN $(20 \mathrm{kHz}$ for 2 minutes) affected the skin penetration of NaFI-loaded PL-LI via the transfollicular pathway. SN significantly decreased the NaFI flux of PL-LI compared to skin without SN. In selectively blocked hair follicles, the skin penetration of NaFI-loaded PL-LI decreased both with and without SN, indicating that the follicular pathway was a major penetration pathway. A CLSM study confirmed that a high intensity of NaFI-loaded PL-LI into the skin and along the length of hair inside the skin was found in skin without $\mathrm{SN}$, while a high intensity on the top of hair outside the skin was observed in the skin with SN. In addition, scanning electron microscopy images revealed that $\mathrm{SN}$ could partially dislocate corneocytes to plug hair follicle orifices; therefore, it could reduce the 

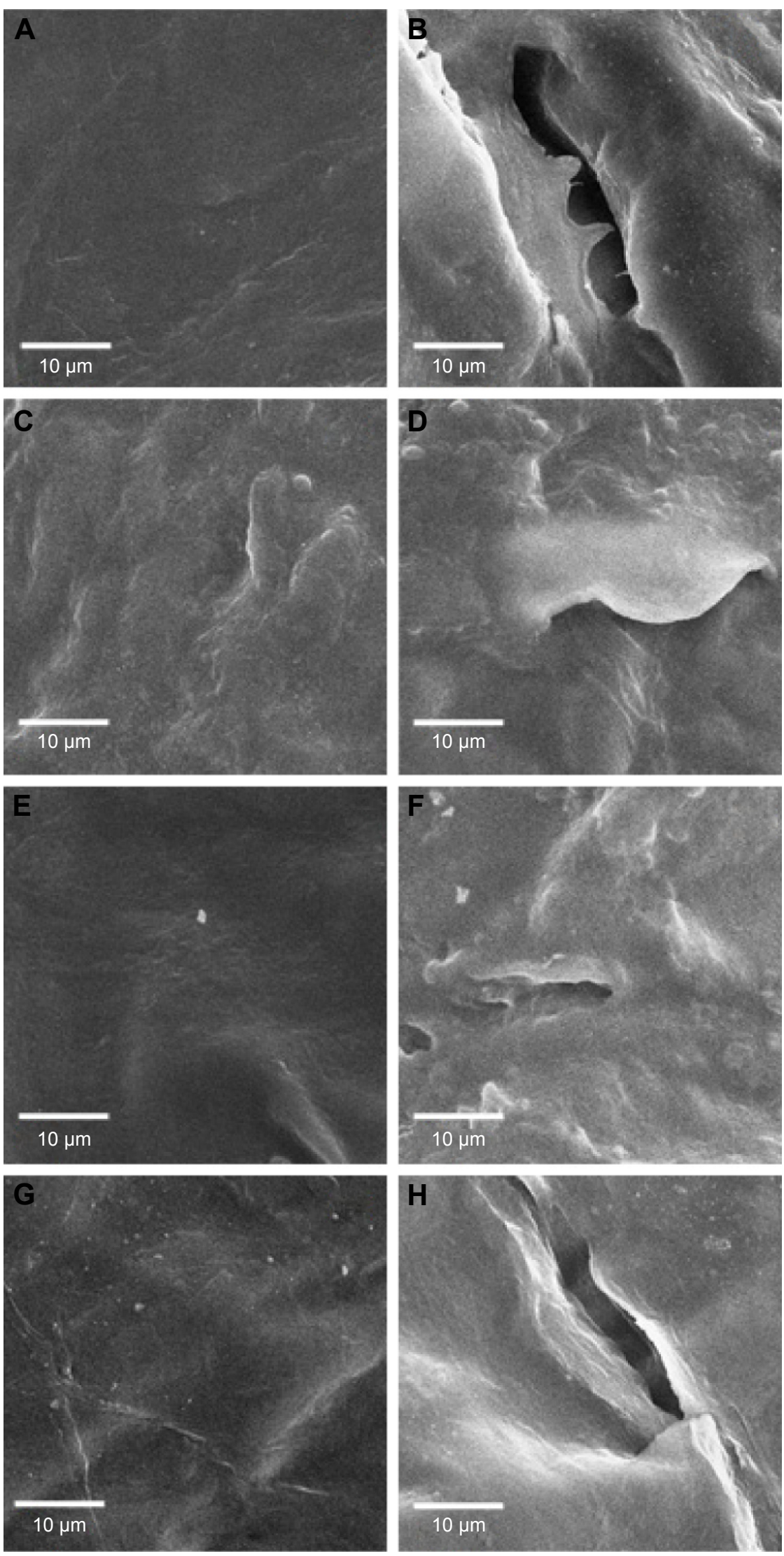

Figure 5 Scanning electron microscopy (SEM) images of porcine skin surface at nonfollicular region (original magnification $\times I, 000)$ ): control (PBS) without SN (A) and with SN (B), CL without SN (C) and with SN (D), PL without SN (E) and with SN (F), and PL-LIs without SN (G) and with SN (H).

Abbreviations: CL, conventional liposome; PBS, phosphate-buffered saline; PL, PEGylated liposome; PL-LI, PEGylated liposome with D-limonene; SN, sonophoresis. 

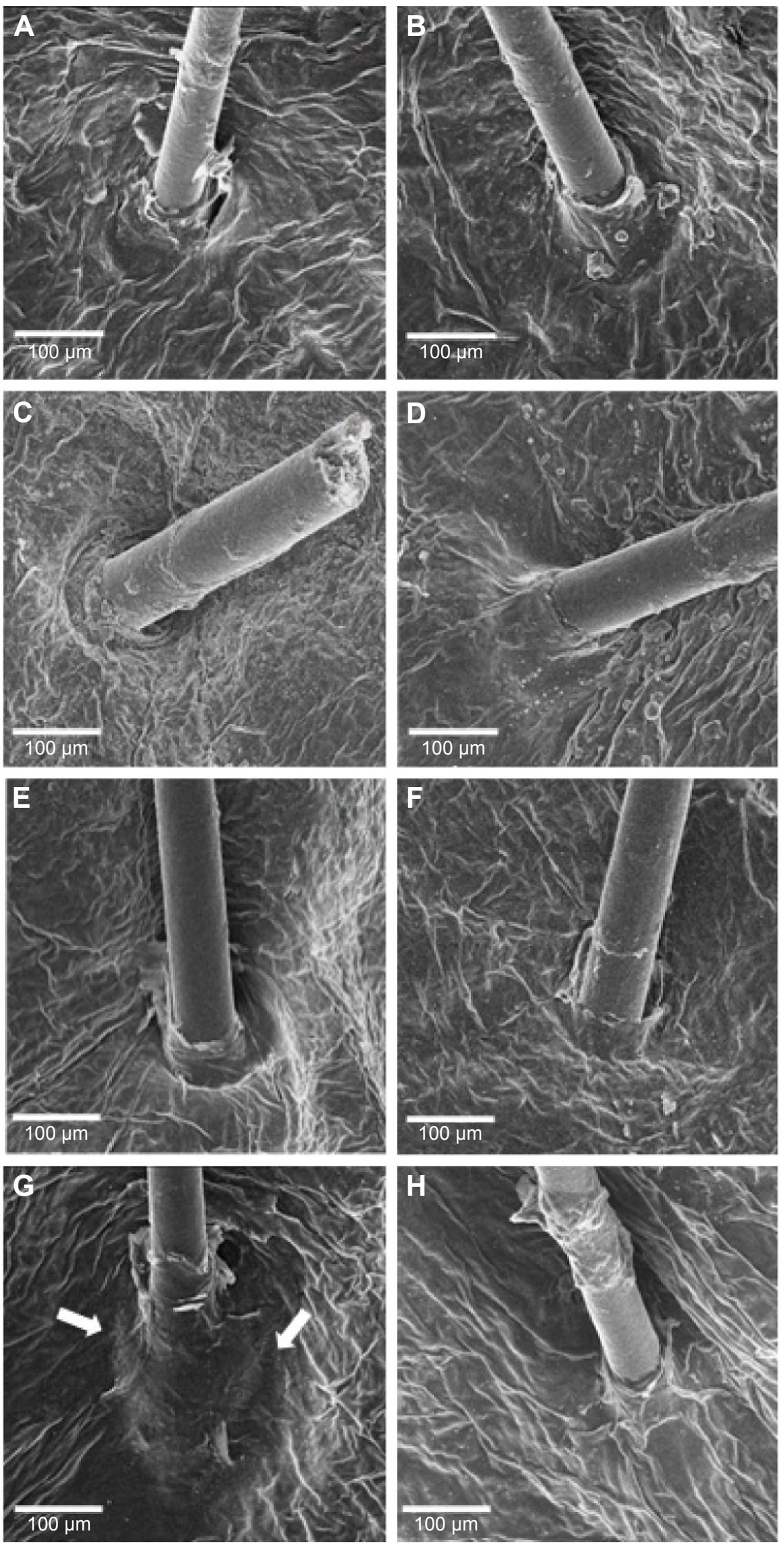

Figure 6 Scanning electron microscopy (SEM) images of porcine skin surface at follicular region (original magnification $\times 120)$ : control (PBS) without SN (A) and with SN (B), CL without $S N(\mathbf{C})$ and with $S N(\mathbf{D})$, PL without $S N(\mathbf{E})$ and with $S N(\mathbf{F})$, and PL-Lls without $S N(\mathbf{G})$ and with $S N(\mathbf{H})$.

Note: The white arrows are PL-LI covered hair follicle orifices (G).

Abbreviations: CL, conventional liposome; PBS, phosphate-buffered saline; PL, PEGylated liposome; PL-LI, PEGylated liposome with D-limonene; SN, sonophoresis. 
percutaneous absorption of NaFI and PL-LI. Therefore, SN might affect the penetration via the follicular pathway.

\section{Acknowledgments}

The authors gratefully acknowledge the Thailand Research Funds through the Basic Research Grant (Grant No 5680016) and the Thailand Research Funds through the Royal Golden Jubilee PhD Program (Grant No PHD/0091/2554) for financial support.

\section{Disclosure}

The authors report no conflicts of interest in this work.

\section{References}

1. Boucaud A, Machet L, Arbeille B, et al. In vitro study of low-frequency ultrasound-enhanced transdermal transport of fentanyl and caffeine across human and hairless rat skin. Int J Pharm. 2001;228:69-77.

2. Sarheed O, Abdul Rasool BK. Development of an optimised application protocol for sonophoretic transdermal delivery of a model hydrophilic drug. Open Biomed Eng J. 2011;5:14-24.

3. Sarheed O, Frum Y. Use of the skin sandwich technique to probe the role of the hair follicles in sonophoresis. Int J Pharm. 2012;423:179-183.

4. Morimoto Y, Mutoh M, Ueda H, et al. Elucidation of the transport pathway in hairless rat skin enhanced by low-frequency sonophoresis based on the solute-water transport relationship and confocal microscopy. J Control Release. 2005;103:587-597.

5. Polat BE, Hart D, Langer R, et al. Ultrasound-mediated transdermal drug delivery: Mechanisms, scope, and emerging trends. J Control Release. 2011;152:330-348.

6. Park D, Park H, Seo J, et al. Sonophoresis in transdermal drug deliverys. Ultrasonics. 2014;54:56-65.

7. Mitragotri S, Blankschtein D, Langer R. Transdermal drug delivery using low-frequency sonophoresis. Pharm Res. 1996;13:411-420.

8. Lee SE, Choi KJ, Menon GK, et al. Penetration pathways induced by lowfrequency sonophoresis with physical and chemical enhancers: iron oxide nanoparticles versus lanthanum nitrates. J Invest Dermatol. 2010;130: 1063-1072.

9. Han T, Das DB. Potential of combined ultrasound and microneedles for enhanced transdermal drug permeation: a review. Eur JPharm Biopharm. 2015;89:312-328.

10. Petchsangsai $\mathrm{M}$, Rojanarata $\mathrm{T}$, Opanasopit $\mathrm{P}$, et al. The combination of microneedles with electroporation and sonophoresis to enhance hydrophilic macromolecule skin penetration. Biol Pharm Bull. 2014; 37:1373-1382.

11. Fang J, Hwang T, Huang Y, et al. Transdermal iontophoresis of sodium nonivamide acetate: V. Combined effect of physical enhancement methods. Int J Pharm. 2002;235:95-105.

12. Mutalik S, Parekh HS, Davies NH, et al. A combined approach of chemical enhancers and sonophoresis for the transdermal delivery of tizanidine hydrochloride. Drug Deliv. 2009;16:82-91
13. Vyas SP, Singh R, Asati RK. Liposomally encapsulated diclofenac for sonophoresis induced systemic delivery. J Microencapsul. 1995;12: 149-154.

14. Dahlan A, Alpar HO, Murdan S. An investigation into the combination of low frequency ultrasound and liposomes on skin permeability. Int J Pharm. 2009;379:139-142.

15. Meidan VM, Bonner MC, Michniak BB. Transfollicular drug delivery is it a reality? Int J Pharm. 2005;306:1-14.

16. Jain S, Tiwary A, Jain N. PEGylated elastic liposomal formulation for lymphatic targeting of zidovudine. Curr Drug Deliv. 2008;5: 275-281.

17. Rangsimawong W, Opanasopit P, Rojanarata T. Terpene-containing PEGylated liposomes as transdermal carriers of a hydrophilic compound. Biol Pharm Bull. 2014;37:1936-1943.

18. Manosroi A, Ruksiriwanich W, Abe M, et al. Transfollicular enhancement of gel containing cationic niosomes loaded with unsaturated fatty acids in rice (Oryza Sativa) bran semi-purified fraction. Eur J Pharm Biopharm. 2012;81:303-313.

19. Kenworthy AK, Simon SA, McIntosh TJ. Structure and phase behavior of lipid suspensions containing phospholipids with covalently attached poly(ethylene glycol). Biophys J. 1995;68:1903-1920.

20. Subongkot T, Wonglertnirant N, Songprakhon P, et al. Visualization of ultradeformable liposomes penetration pathways and their skin interaction by confocal laser scanning microscopy. Int J Pharm. 2013;441:151-161.

21. Patzelt A, Richter H, Buettemeyer R, et al. Differential stripping demonstrates a significant reduction of the hair follicle reservoir in vitro compared to in vivo. Eur J Pharm Biopharm. 2008;70(1):234-238.

22. Jain S, Jain P, Umamaheshwari RB, et al. Transfersomes - a novel vesicular carrier for enhanced transdermal delivery: development, characterization, and performance evaluation. Drug Dev Ind Pharm. 2003; 29(9):1013-1026.

23. Honeywell-Nguyen PL, Bouwstra JA. Vesicles as a tool for transdermal and dermal delivery. Drug Discov Today Technol. 2005;2(1):67-74.

24. Badran M, Shazly G, El-Badry M, et al. Effect of terpene liposomes on the transdermal delivery of hydrophobic model drug, nimesulide: characterization, stability and in vitro skin permeation. Afr J Pharm Pharmacol. 2012;6(43):3018-3026.

25. Alvarez-Román R, Merino G, Kalia YN, et al. Skin permeability enhancement by low frequency sonophoresis: lipid extraction and transport pathways. J Pharm Sci. 2003;6:1138-1146.

26. Merino G, Kalia YN, Delgado-Charro MB, et al. Frequency and thermal effects on the enhancement of transdermal transport by sonophoresis. $J$ Control Release. 2003;88:85-94.

27. Lasic DD. The mechanism of vesicle formation. Biochem J. 1998;256: $1-11$.

28. Grams YY. Influence of Molecular Properties and Delivery System Design on the Transfollicular Transport Across the Skin [dissertation]. Leiden: Division of Drug Delivery Technology of the Leiden/ Amsterdam Center for Drug Research, Leiden University; 2005.

29. Essa EA, Bonner MC, Barry BW. Electroporation and ultradeformable liposomes; human skin barrier repair by phospholipid. J Control Release. 2003;92:163-172.

30. Han T, Das DB. Permeability enhancement for transdermal delivery of large molecule using low-frequency sonophoresis combined with microneedles. J Pharm Sci. 2013;102:3614-3622.
International Journal of Nanomedicine

\section{Publish your work in this journal}

The International Journal of Nanomedicine is an international, peerreviewed journal focusing on the application of nanotechnology in diagnostics, therapeutics, and drug delivery systems throughout the biomedical field. This journal is indexed on PubMed Central, MedLine, CAS, SciSearch ${ }^{\circledR}$, Current Contents ${ }^{\circledR} /$ Clinical Medicine,

\section{Dovepress}

Journal Citation Reports/Science Edition, EMBase, Scopus and the Elsevier Bibliographic databases. The manuscript management system is completely online and includes a very quick and fair peer-review system, which is all easy to use. Visit http://www.dovepress.com/ testimonials.php to read real quotes from published authors. 